\title{
La résurrection du conseil de police au Ghana
}

\author{
Kwesi Aning \\ Directeur, Faculty of Academic Affairs and Research, Kofi Annan \\ International Peacekeeping Training Centre (KAIPTC), Ghana
}

\section{Introduction}

Depuis 1982, le Ghana a connu sa période de stabilité continue la plus longue depuis son indépendance acquise en $1957 .{ }^{1}$ Malgré un périple vers la démocratie interrompu à plusieurs reprises par des invasions militaires (tentatives de coups d'Etat, mutineries et renversements réussis de gouvernements élus démocratiquement), le pays - considéré comme l'Etat ouest-africain le plus mal en point sur les plans économiques et politiques - est devenu après 1981 un véritable modèle de démocratie et de gouvernance du secteur de la sécurité. Dans cette région confrontée à de nombreux défis sécuritaires, certains aspects de cette réussite exemplaire n’ont jamais été traités. La présente étude se propose de remédier à cette lacune en montrant comment les processus de réforme du secteur de la sécurité en ont impacté la dynamique; elle passe en revue quelques uns des changements survenus dans les institutions clés de pouvoir et de gouvernance qui ont été à l'origine de la transformation du pays. Dans cet esprit, l'auteur étudie les effets du rétablissement du Conseil de police au titre de la Constitution de 1992 ainsi que les dysfonctionnements passés de cette institution, qui ont conduit à la situation actuelle dans l'administration des Services de police du Ghana (Ghana Police Service - SPG). Afin de bien comprendre le cheminement logique de cette présentation illustrant les premiers pas vers un vrai contrôle démocratique renforcé du secteur de la sécurité, il est nécessaire d'analyser l'historique des changements graduels qui l’ont engendré.

Comment citer ce chapitre du livre:

Aning, K. 2015. La résurrection du conseil de police au Ghana. Dans : Bryden, A et Chappuis, F (dir. publ.) Gouvernance du secteur de la Sécurité : Leçons des expériences ouest-africaines, Pp. 23-40. London: Ubiquity Press. DOI: http://dx.doi. org/10.5334/bav.b. Licence: CC-BY 4.0. 
L'histoire politique du Ghana et ses diverses péripéties sécuritaires ont façonné les mécanismes et les processus qui permettent aujourd'hui de gérer son secteur de la sécurité. Pour comprendre comment la transformation du secteur s'est opérée, il faut placer les processus de changement dans le contexte mouvementé de la sphère politique postindépendance et celui des progrès réalisés dans les domaines de la gestion et de la supervision. Les institutions de tutelle concernées ont spontanément et pleinement adopté l'idée d'un contrôle démocratique. En conséquence, les manquements et les insuffisances en matière de gouvernance, si typiques des périodes de régime militaire, ont commencé à s’atténuer, bien qu'ils n’aient pas été totalement éradiqués, comme on peut le constater dans le cas du Conseil de police.

Depuis le début des années 2000, le Ghana a progressivement inversé le processus de militarisation qui caractérisait auparavant la scène politique du pays, instituant à la place des processus de gouvernance du secteur de la sécurité relativement stables, lesquels ont permis la mise en place d'une démocratie solide et à lécoute de sa population. Cependant, même si plusieurs de ces institutions de contrôle ont été créées avec l'idée de garantir une gestion démocratique du secteur, celles-ci ne donnent pas encore les résultats escomptés: les SPG et le Conseil de police en sont de bons exemples. ${ }^{2}$

Les Services de police du Ghana ont vu le jour à l'époque précoloniale. L'institution a mué plusieurs fois allant d'une «force» à un «service» afin d’améliorer son efficacité et ses résultats. De ce point de vue, la IIe Constitution républicaine de 1969 a reconnu la nécessité de mettre sur pied un Conseil de police, créé pour la première fois en 1970. Pourtant, de l'avis général, les SPG nont pas fait preuve de l'efficacité voulue et nont jamais pu répondre aux attentes de ceux qu'ils étaient censés servir. Deux raisons majeures expliquent ce mécontentement: leurs ressources humaines sont insuffisantes et leur volonté politique peine à appliquer pleinement le principe du contrôle démocratique par le biais du Conseil de police. ${ }^{3}$

En tant quétude de cas, les SPG et le Conseil de police démontrent comment l'idée même du besoin d'un changement est née, et comment elle a été imposée dans la jungle politique et bureaucratique des différents gouvernements militaires et démocratiques qui se sont succédé, soulignant ainsi les défaillances des organes institutionnels créés. Létude commence par analyser les enjeux stratégiques et historiques durant les changements politiques intervenus dans le secteur de la sécurité au Ghana. Puis, elle entreprend d'examiner les mécanismes qui ont conduit à la résurgence du Conseil de police en 1992, s'attachant plus particulièrement à étudier les principaux acteurs impliqués ainsi que la durabilité de diverses réorientations stratégiques. Pour résumer, le présent chapitre analyse les différentes facettes des cadres juridiques et institutionnels, qui renforcent la gouvernance et permettent le fonctionnement d'un organe majeur du secteur de la sécurité ghanéen, dans le but de définir les meilleures pratiques susceptibles d'inspirer des conceptions élargies de la RSS en Afrique de l'Ouest. 


\section{Le contexte de la gouvernance du secteur de la sécurité au Ghana}

Juste après l'indépendance, alors que le secteur de la sécurité était dirigé par le président Kwame Nkrumah de 1957 à 1966, il a été décidé de créer une garde présidentielle et d'étendre le contrôle exercé par son parti, le Parti de la convention nationale, à l'ensemble du secteur de la sécurité. Les avis sont partagés quant à savoir si ce dernier a perdu ou gagné en appui politique et en crédibilité au cours des régimes suivants, ceux d'Akwasi Amankwa Afrifa (de 1966 à 1969), d'Ignatius Kutu Acheampong (de 1972 à 1978) et de Jerry Rawlings (du 4 juin au ler octobre 1979 et de 1982 à 1992) alors que les motivations et les organes de tutelle et de supervision étaient gravement compromis par des ingérences politiques. En fin de compte, les présidents ont « réussi à politiser subrepticement le leadership des institutions de sécurité de l'Etat, renforçant ainsi l'esprit de parti » (Adu-Amanfo 2014: 99). Ces ingérences dans l'exécution des tâches professionnelles et la prestation de services publics ont atteint leur point culminant avec «l'exigence d'une réciprocité au détriment des seuls mérite et professionnalisme» (Adu-Amanfo 2014: 100). La "politisation excessive des dirigeants des institutions de sécurité a donc provoqué un schisme virtuel dans les allégeances, accentuant ainsi l'esprit partisan parmi les troupes» (Adu-Amanfo 2014: 100) et induisant «une ingérence politique injustifiée dans leur travail quotidien d'administration, de planification et d'exécution des opérations » (Adu-Amanfo 2014:100-102). Certes, l'immixtion politique ne se limitait pas à l'armée et à la police, elle touchait également les milieux du renseignement (Aning, Birikorang \& Lartey 2013: 199-201). Hutchful (1999: 97-118) a qualifié ce processus cumulatif de «mécanisme endémique de militarisation ».

Pourtant, malgré les effets néfastes la performance et la cohésion professionnelles, le Ghana a fini par inverser la tendance. Le retournement a pu se faire grâce aux décisions prises par les différents régimes militaires qui se sont succédé entre 1966 et 1992: le Conseil national de libération, le Conseil de la rédemption nationale, les Conseils militaires suprêmes I \& II et le Conseil provisoire de la défense nationale. Bien quétant une dictature, ce dernier a promulgué, dix ans après son arrivée au pouvoir, la Constitution de 1992 afin de rétablir les principes démocratiques en réponse aux pressions de la population et de la communauté internationale. Ainsi a été instaurée la IVe République. L'inversion de la militarisation s'est intensifiée au cours de cette période sous l'égide du Congrès national démocratique, qui a gouverné entre 1992 et l'an 2000. Entre 2000 et 2008, sous la tutelle du Nouveau parti patriotique, ces multiples changements ont permis d'améliorer le contrôle ainsi que la reprofessionnalisation du secteur de la sécurité et de renforcer le sentiment d'avoir réussi à mettre en place un véritable contrôle démocratique du secteur de la sécurité (Hutchful 1999: 109). ${ }^{4}$ 


\section{La création du Conseil de police}

Le Conseil de police a été mis en place après le rétablissement de l'Etat de droit en 1970: cela a été l'une des premières initiatives du régime du Parti du progrès nouvellement élu et dirigé par Kofi Abrefa Busia. En fait, l'impulsion initiale avait été donnée durant le régime dictatorial du Conseil national de libération juste après le premier coup d'Etat (1966). L'on peut se demander pourquoi un régime noyauté par l'armée et la police, dont les principes démocratiques nétaient pas enracinés, avait inscrit une telle disposition dans sa Constitution de 1969.

Les causes de la création du Conseil de police sont historiques. Juste après l'indépendance, entre 1957 et 1966, alors que le pays était dirigé par Kwame Nkrumah, en raison du climat de méfiance qui régnait entre le Parti de la convention nationale de Kwame Nkrumah et l'armée, il a tout à coup été décidé d'équiper les «forces de police du Ghana » (leur appellation de l'époque) afin de contrebalancer les forces armées: cette mesure de protection visait à prévenir toute éventuelle tentative de renversement du Parti de la convention nationale par l'un des services du secteur de la sécurité. Mais quand le parti de Kwame Nkrumah s'est fait plus autoritaire, restreignant petit à petit l'espace démocratique, une force composée à la fois de policiers et de militaires s'est entendue pour renverser le Parti de la convention nationale le 24 février 1966. Le Conseil national de libération, dirigé par des officiers de la police et de l'armée, a été établi au lendemain de ce coup d'Etat. ${ }^{5}$

L'une des premières initiatives du Conseil national de libération a été de mettre sur pied une campagne pour rappeler à la population ses droits civiques et ses responsabilités. Entre 1966 et l'avènement de la IIe République en 1969, Busia a organisé plusieurs campagnes de sensibilisation dans tout le pays. Par exemple, le Forum d'éducation civique, tribune de la société civile créée par le régime du Conseil national de libération et dirigée par lui-même dans le but d'inculquer des valeurs de bon citoyen à la société ghanéenne. Ce rôle stratégique lui a permis de constater le fossé profond existant entre la création/ disponibilité d'institutions et leur capacité opérationnelle. Par la suite, Busia est devenu premier ministre (1969-1972). C'est grâce à son charisme et son idéalisme que le Conseil de police a pu voir le jour. Il nétait pas seulement un érudit, il était aussi un homme politique: au début de sa carrière, il avait écrit un ouvrage précurseur sur la difficulté de choisir un chef dans la société ghanéenne (Busia, 1951), insistant sur l'importance de maintenir un équilibre dans tous les processus et procédures institutionnels. Parce qu'il avait eu l'expérience du pouvoir politique, il a cherché à joindre la théorie à la pratique en prônant la création d'une institution consacrée à la supervision du travail de la police. La mise en place du conseil a donc été une mesure innovante dont le but nétait pas seulement de contrôler les institutions, mais aussi de tenir le parlement au courant des activités de la police par le biais d'un rapport annuel rédigé par le conseil et débattu au sein du parlement. 
Mettant à profit ses liens étroits avec le régime du Conseil national de libération, lorsque le processus de réintroduction de la politique démocratique a été enclenché en 1968, Busia et ses acolytes ont formé le Parti du progrès, qui a par la suite remporté les élections de 1969. Après leur entrée en fonction sous l'appellation «IIe République», l'une de leur réorientation politique la plus remarquable a été l'adoption de la loi de 1970 sur les services de police (loi no 350) inspirée de la Constitution de 1969. C’est l'instrument législatif le plus important qui régit le secteur de la sécurité, notamment les SPG, avec leur système de séparation des pouvoirs, leur réglementation et leurs codes de fonctionnement complexes. ${ }^{6}$ La loi no 350 a été promulguée en vue d’améliorer l'efficacité et la supervision de l'ensemble des services de police et de permettre au parlement d'exercer un contrôle démocratique. Elle comporte à cet effet toute une série de dispositions et de règlements décrivant en détail les différents volets de ses fonctions de gestion et de supervision. Lorsque le Conseil de police a été formé en 1970, il était composé de dix membres et était l'organe constitutionnel chargé de conseiller le président à propos des questions stratégiques relatives à la sécurité intérieure, y compris le rôle et la capacité opérationnelle des SPG. ${ }^{7}$ Cette initiative a marqué une évolution importante et novatrice, sans doute imputée en partie au fait que les dirigeants de la IIe République avaient travaillé en étroite collaboration avec la direction militaro-policière du Conseil national de libération, ce qui fait qu'ils comprenaient particulièrement bien l'importance d'instituer un contrôle et savaient aussi comment en faire accepter l'idée par le secteur de la sécurité et par la population en général.

La loi no 350 comporte plusieurs articles importants. En premier lieu, elle stipule que l'inspecteur général de la police et, par extension, les SPG doivent soumettre des rapports annuels au parlement par l'intermédiaire du Conseil de police:

Aussitôt que possible après le 30 juin de chaque année, l'inspecteur général de la police devra préparer un rapport décrivant en détail l'activité de ce service ... au cours des douze mois écoulés. Le rapport sera soumis au ministre qui le présentera à l'assemblée nationale. ${ }^{8}$

En vertu de la loi no 350, les fonctions du Conseil de police en tant qu'organe consultatif statuant sur les nominations, le bien-être, la discipline, le recrutement, la formation, les relations entre la police et la population ainsi que sur les décisions relatives aux recours en appel disciplinaire émanant de militaires en activité. ${ }^{9}$ Outre l'obligation légale de présenter un rapport au parlement, le conseil devra:

- réviser les obligations de contrôle constitutionnelles et légales des services de police;

- évaluer dans quelle mesure les missions ont été menées à bien;

- présenter les raisons expliquant leurs succès et leurs échecs; 
- déterminer les mesures à mettre en place pour remédier aux défaillances opérationnelles des services; et

- convenir de mesures pragmatiques, de programmes et d'activités visant à renforcer le rôle de supervision du conseil.

Avec l'aval du président, le conseil peut également proposer des dispositions réglementaires susceptibles d'améliorer l'administration des services de police. ${ }^{10}$

\section{L'atrophie sous les dictatures militaires}

Malgré létablissement du Conseil de police par le régime du Parti du progrès, l'obligation formelle de tenir le parlement informé au moyen de rapports annuels n’a jamais été respectée. D’aucuns pourraient avancer, quà l'époque, le conseil n'avait que peu de chances d'arriver à mettre au point et instituer ses procédures de travail avant qu'un régime militaire ne réapparaisse. Larmée est revenue au pouvoir en 1972 après un coup d'Etat orchestré par Ignatius Kutu Acheampong, qui a alors créé le Conseil de la rédemption nationale, luimême suivi en 1978-1979 du second Conseil suprême militaire de Frederick William Kwasi Akuffo. Bien qu'Acheampong et Akuffo aient tous deux été des officiers de l'armée, il semble qu'ils aient eu une certaine affinité avec la police, ce qui explique que cette dernière a bénéficié d'un soutien logistique et d'autres avantages en équipements bien plus importants que ceux attribués aux forces armées. Plusieurs services de la police ont été constitués, et les SPG ont été renommées «forces de police du Ghana». Ce changement d'appellation de «services» en "forces» était la preuve que les nouveaux dirigeants militaires avaient l'intention de doter l'institution policière de la puissance nécessaire pour à la fois contenir la population en cas de troubles et contrer les velléités des militaires.

Qui plus est, en 1974, le décret (amendement) no 303 sur les forces de police (NRCD 303) a apporté des modifications à certains articles de la loi no350. Ce changement était primordial car les SPG ont été dispensés du contrôle administratif et bureaucratique de la Commission des services publics. Autre changement majeur: l'inspecteur général de la police a été habilité à présider son propre Conseil de police et élevé au rang de ministre. Une mesure aussi radicale signifiait que le juge devenait partie, empêchant ainsi tout contrôle efficace et accordant une autorité pratiquement sans limites à l'inspecteur général de la police.

Ainsi, entre le coup d'Etat de 1972 et 1992, il y a eu un vide démocratique, les institutions démocratiques du pays étant phagocytées par un dictateur après l'autre. La création du Conseil de police a été l'exception, sans doute parce qu'il était le fruit des convictions idéalistes d'un érudit. Cependant, les interventions récurrentes de l'armée dans la politique interrompaient sans cesse le travail du conseil. Pour comprendre ces événements, il faut savoir que le secteur 
de la sécurité ghanéen a été régi depuis toujours par un réseau complexe de réglementations, dont certaines étaient parfois temporairement abandonnées, tandis que d’autres étaient négligées, abrogées ou laissées en attente au gré du régime de lépoque. Lorsque le cadre juridique régissant le pays a été mis en suspens après les coups d'Etat qui ont donné naissance à de nouvelles institutions coercitives, celles qui étaient déjà actives dans le secteur de la sécurité n’ont pas osé contester le nouveau régime, pour le moins parce qu'elles nétaient pas certaines de connaître à quel niveau de la chaîne de commandement les nouveaux ordres avaient été donnés. Le problème s'est surtout posé lorsque de nouvelles modalités de prise de décision élaborées lors de régimes militaires cherchaient à saper le bon fonctionnement des institutions existantes. ${ }^{11}$ Ainsi, pendant ces périodes, lorsque la constitution en vigueur était en suspens, les forces armées et les SPG ont continué à respecter leurs règlements et procédures administratives ou disciplinaires internes. Il faut cependant noter qu'ils ont été maintenus non seulement pour des impératifs de hiérarchie et de contrôle mais aussi, dans le cas des SPG, pour donner une impression de continuité. Le Conseil de police - organe normalement responsable de l'application de ces règlements intérieurs conformément aux exigences en matière de contrôle démocratique et d'obligation de rendre compte - a été laissé à l'abandon et s'est affaibli. Ce n'est qu'au terme d'une longue parenthèse, entre 1979 et 1992, quand la règle constitutionnelle a été rétablie, qu'il a connu un renouveau sous la $\mathrm{IV}^{e}$ République.

$\mathrm{Si}$ le Conseil de police est devenu ce qu'il est aujourd'hui, c'est parce que l'espace politique s'est progressivement ouvert et que d'autres institutions non conventionnelles dites « sécurocratiques» s'y sont elles aussi invitées pour aider à refondre le secteur (Aning 2008b). Petit à petit, le secteur de la sécurité du Ghana sest mis à adopter des processus de gouvernance plus participatifs et plus transparents. L'on a ainsi :

une bonne idée de ce quest la gouvernance du secteur de la sécurité en Afrique de l'Ouest, ne serait-ce que parce qu'un processus de militarisation apparemment endémique semble avoir été stoppé et même inversé vers la fin des années 80 et des années 90, facilitant une transition vers une démocratie relativement stable. Et s'il est vrai quà certains égards le cas du Ghana est représentatif d'un grand nombre de transitions à partir d'un autoritarisme militaire vers une démocratie électorale en Afrique de l'Ouest et qu'il a en commun avec elles maintes contraintes et ambigüités, il se démarque cependant par son véritable élan démocratique. L'évolution régulière de la gouvernance du secteur de la sécurité en a été un élément essentiel (Hutchful 2004:1-2)

Dans son analyse, Hutchful montre pourquoi la transition réussie du pays à partir de régimes militaires principalement autocratiques juste après l'indépendance en 1957 vers l'instauration de la IV ${ }^{e}$ République en 1992 est si extraordinaire. Malgré la diversité des gouvernements en place durant cette période et 
malgré le nombre de changements et de programmes qui ont façonné le secteur de la sécurité, les styles de direction et de gestion ont généralement été marqués par un certain pragmatisme. Avant la IIe République en 1970, il y avait déjà eu des tentatives pour réformer, approfondir et renforcer les systèmes ghanéens de gouvernance démocratique en matière de sécurité, et des mécanismes de contrôle institutionnels de plus en plus efficaces se sont graduellement imposés.

Pour comprendre comment fonctionne le secteur de la sécurité au Ghana, il faut considérer conjointement les attentes de la population pour une réforme sectorielle et le rôle des institutions bilatérales et multilatérales. En fait, la réforme permettait à la plupart de ces institutions internationales de «fournir un minimum de services publics au moindre coût»: d'importants efforts ont donc été déployés pour renforcer l'efficacité et l’obligation de rendre compte dans les ministères, les services gouvernementaux et les agences officielles, étape essentielle vers une amélioration globale dans le secteur public (Brzoska 2002:1). Malgré l'insistance des partenaires internationaux pour réformer le secteur de la sécurité dans le cadre général des réformes du secteur public, entre 1982 et 1992, l'administration Rawlings s'est montrée réticente à coopérer tout au long de la série des refontes entamées au début des années 80 . Ces réformes du secteur public, qui ont bénéficié d'un financement considérable de la part de la communauté internationale, ont abouti à la création du Programme national pour le renouveau institutionnel, mais excluaient la plupart des institutions de sécurité. ${ }^{12}$ Ce neest que depuis 1996 que l'idée de devoir procéder à une réforme complète des institutions du secteur de la sécurité a gagné du terrain. Elles en ont peut-être encore plus besoin que le reste du secteur public.

\section{La résurgence du Conseil de police}

En 1992, après une longue période de troubles en vue de réinstaurer la gouvernance démocratique, il a été décidé d'entreprendre une révision de la constitution. De ce fait, l'espace politique s'est ouvert et une assemblée constituante a été chargée d'élaborer un nouveau texte. Après la mise en place de mécanismes de gouvernance démocratique à loccasion de la création de la $\mathrm{IV}^{\mathrm{e}}$ République, l'idée d’un Conseil de police a été réintégrée. Ainsi, la $\mathrm{IV}^{\mathrm{e}}$ Constitution républicaine de 1992 a imposé la réintroduction du Conseil de police en vertu de l'article 203, afin de conseiller le président sur les sujets relatifs à la sécurité intérieure, y compris les conditions de travail du personnel des services de police, budgétisation, retraites, salaires et allocation des fonds pour une gestion efficace et rationnelle des SPG. ${ }^{13}$

Ainsi, de même que dans la première mouture, le Conseil de police reconstitué est, de par la loi, un organe consultatif à la disposition du président. Bien que les SPG soient à l'heure actuelle considérés comme étant sous la responsabilité administrative du ministre de l'Intérieur (ils font à présent partie du conseil), 
le Conseil de police lui-même est un organe indépendant. Au niveau du siège, il comporte deux bureaux consultatifs: le Conseil consultatif de la police pour les nominations et les promotions et le Conseil dadministration de la police. ${ }^{14}$ Tandis que le premier formule des recommandations de nominations et de promotions à tous les niveaux, le second est chargé des grandes questions et décisions relatives à l'administration générale des services et aux opérations. Tous deux dépendent du Conseil de police; par conséquent toutes les activités des SPG relèvent de sa compétence et de sa responsabilité. Reste à déterminer si ces organes ont assez d'autorité pour s'acquitter correctement de leurs fonctions. Malgré ces problèmes, tous les observateurs enclins à critiquer le secteur de la sécurité au Ghana s'accordent à penser que le Conseil de police doit continuer à exister et que sa contribution est la bienvenue. ${ }^{15}$

La nouvelle constitution a apporté des changements particulièrement importants dans la composition du Conseil de police, intronisant le vice-président au conseil puis le chargeant de diriger les débats. ${ }^{16}$ Conformément aux nouvelles dispositions, le Conseil de police se compose à présent des membres suivants:

1. le vice-président, qui présidera les séances;

2. le ministre de l'Intérieur;

3. l'inspecteur général de police;

4. le procureur général ou son représentant;

5. un avocat désigné par l'Ordre des avocats du Ghana;

6. un représentant de l'Association des hauts-fonctionnaires de police à la retraite;

7. deux membres des services de police, nommés par le président, travaillant en coordination avec le Conseil d'Etat, dont l'un sera de rang subalterne;

8. deux autres membres désignés par le président.

Ces changements ont été révélateurs d’autres plus importants encore dans le pays en général et dans les SPG en particulier. D’abord, la décision d'intégrer au conseil des membres de l'Association des hauts-fonctionnaires de police à la retraite, a rendu ainsi hommage à leur immense savoir-faire et a montré combien leur contribution était précieuse. Ensuite, l'initiative de faire participer un officier de police de rang subalterne afin de mettre en avant les problèmes rencontrés à ce niveau. Cependant, il n'est pas certain que cette dernière ait eu l'effet escompté car la nature même de la hiérarchie et la tradition militaire font qu'il est pratiquement impossible pour un officier subalterne d'exposer les préoccupations de ses collègues sans craindre des répercussions. ${ }^{17}$ Ce geste symbolique peut être vu comme un héritage de la Révolution de 1981, durant laquelle les subalternes ont brièvement pris le contrôle du gouvernement.

La création de comités de police régionaux a également été prévue dans la nouvelle législation afin de reproduire certaines des fonctions consultatives du Conseil de police à une moindre échelle. Mais ces organes nont jamais vu le jour officiellement. En fait, tous les changements relatifs au Conseil de police 
survenus depuis les progrès accomplis par Busia ne sont que des réglages et ajouts techniques.

Malgré son potentiel et les bonnes intentions qui ont inspiré sa création, le Conseil de police continue à souffrir d'un long passé d'assujettissement au contrôle présidentiel. Par exemple, le poste de vice-président agissant en qualité de président de séance a été révoqué au lendemain de la crise qui aurait pu dégénérer entre le président Jerry Rawlings et feu le vice-président Ekow Nkensen Arkaah. ${ }^{18}$ Bien que la situation se soit un peu améliorée dans la mesure où ledit poste a été rétabli depuis lors, ${ }^{19}$ même en ces temps démocratiques, la composition du Conseil de police continue de favoriser le pouvoir exécutif et n'est toujours pas indépendante. Sur dix membres, huit sont désignés par le président, à l'exception du membre de l'Ordre des avocats et du représentant des hauts-fonctionnaires de police à la retraite. Idéalement, les trois branches du gouvernement devraient être représentées et il devrait également y avoir une représentation de la société civile de façon à mieux prendre en compte les attentes de la population. Au lieu de cela, les membres du conseil sont généralement perçus comme étant sous la coupe du président. En outre, depuis 1970, la plupart des conseils travaillent et prennent leurs décisions par consensus, ne laissant que très rarement le contenu de leurs délibérations parvenir jusquau public.

Le Conseil de police est généralement perçu de l'extérieur - surtout par les membres des SPG - comme une entité dont les membres sont très préoccupés par les "promotions, les rétrogradations et les licenciements $»{ }^{20}$ Pourtant, et bien que ce commentaire soit volontairement dérogatoire, ces mécanismes sont indispensables pour faire respecter l'obligation de rendre compte et garantir un certain sérieux professionnel. Ainsi, la crédibilité du conseil a été mise en cause, notamment à loccasion de promotions apparemment injustifiées ayant provoqué de la rancœur parmi les troupes. Durant ces vingt dernières années, il est parfois arrivé qu'il prenne des décisions arbitraires. Par exemple, à lépoque du gouvernement Kufuor en 2001, un grand nombre de hauts-fonctionnaires de police (y compris l'inspecteur général d'alors) qui avaient atteint l'âge officiel de la retraite ont été priés de partir. Ils occupaient encore des postes élevés car certains avaient vu leur contrat reconduit par le gouvernement, et d'autres étaient restés pour diverses raisons. Bien que cette décision ait été conforme à la loi, ainsi quà la réglementation et aux procédures en vigueur dans les SPG, beaucoup étaient mécontents. ${ }^{21} \mathrm{~A}$ première vue, cette décision semblait être une démonstration de force de la part du conseil, mais ce dernier a fini par s'incliner devant le pouvoir exécutif. De même, en 2003, à l'instigation du conseil, un haut-fonctionnaire de la police avait été promu de préférence à de nombreux officiers supérieurs, ce qui a provoqué une certaine agitation dans les rangs des SPG. Ceci n'est qu'un cas parmi tant d'autres, de promotions et nominations surprenantes. Il y a aussi eu la nomination d'un officier à la retraite au poste d'inspecteur général de la police alors que la candidature d'officiers d'active n’avait même pas été envisagée. Le Conseil consultatif chargé des nominations 
et des promotions prétend qu'il n’a pas été systématiquement consulté. ${ }^{22}$ Si c'est le cas, il faut se poser la question de savoir qui influence le conseil et comment ces décisions sont prises.

Juste après l'inauguration du Conseil de police en novembre 2013, le vice-président Kwesi Bekoe Amissah-Arthur chargé d’en diriger les débats, a déclaré qu'une nouvelle procédure serait mise en place pour la nomination des inspecteurs généraux de police afin déviter les dissensions qui avaient surgi auparavant à ces occasions: la raison en était surtout les querelles politiques au sein des SPG qui entraînaient des tensions dans toute la hiérarchie (Joy Online 2013a). Mais quelques semaines avant la déclaration publique du vice-président, le président a renouvelé le mandat de l'inspecteur général en place sans consulter le conseil, provoquant la consternation dans les services de police (Joy Online 2013b).

A l'heure actuelle, il existe deux obstacles à l'amélioration de la situation et tous deux trouvent leur origine dans la constitution. En premier lieu, les modalités de nomination s'appliquant à l'inspecteur général et aux membres du Conseil de police soumettent les SPG aux caprices du pouvoir exécutif sans qu'il y ait de véritable contrôle démocratique. Les travaux du conseil ne peuvent être perçus comme placés sous la supervision de l'exécutif car ils risqueraient dêtre détournés à des fins politiques. Les SPG sont censés être au service de la population qui doit pouvoir se faire une idée précise de la façon dont les services sont organisés et contrôlés. En second lieu, la fonction d'inspecteur général de police est considérée comme très importante en terme d'influence. Alors que ce poste, perçu comme bénéficiant du soutien tacite de l'exécutif, existe principalement pour des raisons opérationnelles, un Conseil de police faible sera incapable de garantir la séparation des pouvoirs, surtout quand les SPG fonctionnent en tant qu'institution unifiée dans la chaîne de commandement. Dans la pratique, le bureau de l'inspecteur général décide de ce que le Conseil de police doit savoir et des décisions qu'il pourra prendre: tant que le conseil a l'impression que le pouvoir exécutif avalise son action, toutes les propositions seront adoptées, même s'il y a opposition. En fin de compte, l'activité du conseil dépend totalement de l'exécutif, d’autant plus que c'est lui qui nomme la majorité de ses membres. Tous les officiers connaissent les faiblesses et les difficultés qui entravent l'action des SPG, mais peu font entendre leur voix pour dénoncer ces insuffisances flagrantes. Jadis, il y avait davantage de plaintes, alors que la situation était bien meilleure quà présent.

\section{Pour un changement durable}

Dans le cas du Ghana, les grands changements politiques relatifs au Conseil de la police avaient été initiés par plusieurs intervenants influents. Busia, à la fois un universitaire, un défenseur des droits de l'homme et un chef de l'opposition de longue date, a su tirer profit de son étiquette de démocrate pour 
institutionnaliser des processus plus transparents. Bien qu'il ait été l'origine du Conseil de police, il n’a pu en assurer le bon fonctionnement, le gouvernement l'en ayant empêché et ayant fait valoir ses attentes. ${ }^{23}$ Depuis l'avènement de la IVe République, les contraintes habituelles auxquelles le Conseil de police était confronté - notamment le manque de ressources humaines et financières et l'absence de contrôle de l'exécutif - ont été compensées par les opportunités, de plus en plus fréquentes, délargir le savoir-faire de la société civile et de renforcer sa participation au sein du secteur de la sécurité, même si ces opportunités sont encore trop souvent contradictoires. Toutefois, les progrès accomplis dans ce domaine ont permis d'améliorer les capacités et de stimuler l'intérêt de partis politiques et de membres du parlement pour les questions sécuritaires. Juste après la période autoritaire, en 1992, les relations entre le parlement/les partis politiques et le secteur de la sécurité étaient profondément marquées par la méfiance ${ }^{24}$ mais lon constate que, depuis le début de la IVe République, des efforts ont été déployés afin délargir la portée de la réforme, y compris en matière de sécurité.

Si lon étudie l'efficacité du Conseil de police du point de vue de sa mission, on constate qu'il existe une étroite corrélation entre l'efficacité/l'obligation de rendre compte des SPG considérés dans leur ensemble et les résultats obtenus par le conseil. Dès lors que le conseil est perçu comme étant un organe de terrain travaillant en étroite collaboration avec l’inspecteur général et léquipe de gestion administrative basée au siège, la performance, l'efficacité et la prestation de services des SPG s’améliorent (Salia 2015).

Dans l'ensemble, il reste encore du chemin à parcourir si l'on veut améliorer les prestations et le fonctionnement des SPG et du Conseil de police. Pour aller de l'avant, les SPG doivent se fixer des objectifs clairs et établir des normes; ils doivent décider d'un programme indiquant les grands thèmes à traiter en priorité ainsi que les procédures opérationnelles permanentes à mettre en place sur les plans de la gestion et du fonctionnement. Il existe des stratégies, mais leur mise en œuvre manque de cohérence, y compris pour les critères d'admissibilité pour le recrutement. Officiellement, la police respecte ces exigences, mais lon a pu constater ces derniers temps une tendance au «protocolisme» de la part de personnalités influentes telles que des membres du gouvernement, qui ont commencé à diffuser une certaine incompétence à travers toutes les institutions étatiques ghanéennes. Les réglementations et les lois doivent être renforcées par le Conseil de police ainsi que le prévoit l'article 203(2) de la Constitution de 1992 de façon à pouvoir être appliquées à l'ensemble des SPG. Le conseil doit donner du poids aux réglementations, aux lois et procédures pertinentes pour éviter que les progrès accomplis ne perdent de leur légitimité. De même, si les nominations au conseil gagnent en transparence sans être assorties des améliorations correspondantes dans les SPG, tout ceci ne serait qu'une façade: les deux termes de léquation sont interconnectés et entremêlés à tous égards. 
La transparence est primordiale en ce qui concerne les questions budgétaires. Le gouvernement actuel semble concentrer ses efforts sur la création d'unités de police constituées : des équipements ont été reçus et 170 officiers seront bientôt envoyés en mission au Soudan du Sud. ${ }^{25}$ Néanmoins, le Conseil d'administration de la police lui-même ignore la source du financement de ces équipements. Si lui, qui est censé aider le Conseil de police à prendre les décisions stratégiques, n'est pas au courant, alors qui le sera? C'est d'autant plus important que lorsque les moyens manquent, les capacités des SPG s'en ressentent fortement. Les véhicules ne sont pas entretenus et certaines unités ou postes ne disposent pas des effectifs requis ni de l'équipement nécessaire pour s'acquitter des tâches. La stratégie en vogue, privilégiant la mise en place d'agents de police là où ils sont bien visibles, a éloigné de leurs postes des officiers compétents pour les placer aux carrefours, tandis que d'autres missions essentielles sont mal exécutées, voire pas du tout. Ne serait-ce quà Accra, on estime qu'environ 1000 officiers sont affectés quotidiennement à ces tâches alors qu'il manque des inspecteurs et des officiers pour remplir les fonctions de première nécessité. Cela a des répercussions dans tous les quartiers, départements et régions. ${ }^{26}$ Le faible nombre de cas résolus en proportion du nombre élevé des cas faisant l'objet d'une enquête signifie que les prisons du pays sont pleines d'individus en détention préventive. Si le Conseil de police insistait davantage auprès de l'inspecteur général et de l'équipe chargée des enquêtes criminelles, ce problème pourrait être atténué. $^{27}$

Un autre échec imputable au conseil est la nomination de l'inspecteur général de la police et d'autres membres importants du personnel de l'administration centrale. Les descriptions de postes et les instructions telles quelles existent aujourd'hui devront être revues de façon à permettre la supervision et lévaluation de ces personnes en fonction de critères précis. Ce serait là une façon de garantir non seulement l'application des lois, procédures et protocoles pertinents, mais aussi la conformité avec les exigences à satisfaire. D'ailleurs à ce sujet, il faudra sensibiliser les troupes à la culture des SPG, qu'ils ne comprennent pas. Etant donné que ces derniers transmettent leurs décisions aux commandements, aux unités et aux troupes par le biais d’ordres de mission, d'instructions, de signaux et de circulaires, les Conseils d'administration de la police pourraient utiliser ces mêmes canaux pour expliquer les énoncés de mission et la doctrine des SPG. Le système existant serait ainsi mis à profit pour diffuser des informations et documents pertinents depuis le haut de la chaîne de commandement vers le bas, ce qui permettrait d’établir une cohérence et de bonnes bases pour évaluer l'efficacité de l'ensemble des SPG.

Pour résumer, la réintégration du Conseil de police durant la $\mathrm{IV}^{\mathrm{e}}$ République a représenté un changement de politique majeur et durable en ce qu'il a permis de rétablir le contrôle des SPG. Le conseil continue d'être considéré comme une institution nécessaire dont les perspectives à long terme permettent d'espérer une amélioration de l'efficacité des SPG, même s'il reste encore beaucoup à faire. 


\section{Conclusion}

Inscrire ces événements dans une perspective historique permet de mieux comprendre la situation particulièrement délicate du Conseil de police, dont l'analyse reste fragmentaire, ainsi que les conséquences plus générales sur la gouvernance du secteur de la sécurité. Dans l'ensemble, le contrôle démocratique de ce secteur au Ghana s’est amélioré depuis les années 90. Néanmoins, l'héritage du contrôle militaire se fait encore sentir. Ainsi, le pouvoir du parlement reste limité par rapport à celui de l'exécutif. Il faudra renforcer sa capacité institutionnelle afin de rétablir léquilibre et lui permettre d'exercer ses fonctions de supervision prescrites par la loi: il faudra également lui allouer les ressources nécessaires pour mettre en œuvre ces changements de manière durable. En outre, certains volets de la législation en vigueur, adoptés pour réglementer les activités et les prestations des principales institutions du secteur de la sécurité, ont fini par restreindre le contrôle démocratique au lieu de l'encourager: il faudra les réviser et les modifier. Les mutations fréquentes de personnel et la "politicisation» excessive des grandes institutions de supervision et de contrôle ont affaibli une capacité en ressources humaines déjà mal en point. Même si toutes ces questions étaient résolues, pour être véritablement efficaces, les activités des institutions de contrôle telles que le Conseil de police devraient être complétées par une participation plus étoffée de la part de la société civile. La société civile ghanéenne doit jouer un rôle plus actif dans les réseaux, les débats et les campagnes de sensibilisation si le pays veut élargir son espace démocratique, que ce soit au niveau du secteur de la sécurité ou à un niveau plus global.

Ce n’est quà partir de 1992, que les processus parlementaires ont commencé à avoir un impact sur le secteur de la sécurité. L'incapacité chronique du parlement à exiger un rapport annuel de la part du Conseil de police montre qu'il y a encore beaucoup à faire, même si des progrès considérables ont été accomplis en ce qui concerne la législation et les dispositions constitutionnelles régissant le secteur de la sécurité. Les efforts déployés par les différents parlements qui se sont succédé ont été entravés par la rareté des études disponibles sur le thème de la sécurité, par l'influence des partis et par le renouvellement des membres du parlement du fait des élections. Un Conseil de police efficace et opérationnel, assorti d'un contrôle parlementaire approprié, doit être guidé par le pragmatisme et le réalisme politique plutôt que par les intérêts de partis ou d'individus. Malgré le très faible nombre détudes traitant du Conseil de police et des services de sécurité au Ghana, on peut tirer plusieurs conclusions générales. Tout d'abord, il faut s'efforcer de mieux concilier la théorie avec la pratique. Même si les textes de loi reconnaissent explicitement le rôle que le conseil peut jouer, ce rôle vidé de sa substance est néanmoins mis en exergue à chaque déclaration officielle quand il s'agit de porter les valeurs institutionnelles devant la société civile et le reste du monde. Ensuite, alors qu'il est investi d'importantes responsabilités stratégiques et de tâches à accomplir, le conseil 
n’a généralement rien fait d'autre que découter les griefs des officiers, esquivant les missions plus délicates comme celle de formuler des recommandations stratégiques ainsi qu'il est stipulé dans la constitution. Nonobstant sa faiblesse actuelle, la scène politique et la société civile du Ghana conviennent du fait que, s'il est bien dirigé et dispose de suffisamment de moyens, le conseil peut être (et sera) utile dans le cadre des fonctions de supervision et de réglementation, comme cela a été prévu par les rédacteurs de la Constitution de 1969 et la loi sur les services de police de 1970. Cependant, pour que les progrès soient durables, il faudra renforcer les fonctions et pouvoir officiels du Conseil de police de façon à ce que les décisions ne puissent pas être annulées par l'exécutif ou du fait du remplacement de ses membres.

Quelles sont les conséquences de tout ceci pour les réformes axées sur la gouvernance en Afrique de l'Ouest? Au Ghana, la rhétorique n'est pas toujours étayée par le comportement des institutions, à savoir leur indépendance, leur motivation et leur efficacité; la capacité du pays à lancer des réformes axées sur la gouvernance et à les faire durer s'en trouve entravée. Plus grave encore, on constate dans toute la région ouest-africaine que l'enthousiasme et l'optimisme initiaux du début des années 2000 pour mettre en place des réformes se sont transformés en léthargie, et que parfois même il y a eu un retour en arrière par rapport aux progrès accomplis.

Trois questions restent en suspens. Premièrement, comment favoriser des changements politiques de façon à améliorer l'efficacité du Conseil de police? Deuxièmement, comment faire perdurer ces changements? Troisièmement, comment renforcer de manière plus efficace et durable la capacité en ressources humaines afin de stimuler l'intérêt des intellectuels et de la société civile? Les universitaires, en particulier, ont un rôle à jour non seulement pour créer du savoir, mais aussi pour en encourager l'accès à d'autres parties prenantes et permettre l'expression d'opinions autres que celles des SPG. Cependant, à l'heure actuelle, il est difficile d'étudier les travaux du Conseil de police et d'en discuter car, excepté quelques brefs messages communiqués à la sortie de réunions officielles, il n'existe aucun document écrit qui fasse état de ses processus de prise de décision ni de la nature des débats. Pour améliorer le dialogue entre la société civile et le conseil, il faudra des initiatives originales allant puiser à l'extérieur les compétences requises pour enrichir et renforcer la base de connaissances.

Le cadre institutionnel et législatif du Ghana s'efforce de créer un environnement propice à la gouvernance démocratique, notamment au sein du secteur de la sécurité. Mais, étant donné que les lois sont de moins en moins respectées, dans l'attente d'élections controversées, peut-être même violentes, en 2016, lavenir est incertain. Pour garantir la pérennité des processus démocratiques, il est urgent de raviver lethos qui a inspiré la création du Conseil de police et de se concentrer sur la mise en place de services policiers dynamiques et à lécoute, capables de résister dans un contexte de plus en plus hostile. Plus les processus et mécanismes démocratiques se renforcent au Ghana, plus les 
travaux du conseil se révéleront utiles: son orientation stratégique peut et doit jouer un rôle crucial tandis que les SPG luttent pour répondre aux attentes de la population.

S’il reste des défis à relever, cela ne diminue en rien le mérite du pays d'avoir surmonté l'instabilité politique des années 70 et 80 et d'avoir réinstauré ses institutions démocratiques, dont le Conseil de police fait partie. Le Ghana aura au moins réussi à mettre en place un semblant de contrôle des services de police, et c'est là une réussite remarquable et pleine de promesses.

\section{Notes}

1 Je tiens à remercier mes collègues, et en particulier Nana Bemma Nti et Paul Avuyi, de la faculté des affaires académiques et de la recherche du Centre international Kofi Annan de formation aux opérations de maintien de la paix pour mavoir aidé de leurs précieux conseils lors de la rédaction des premières versions de cette étude. Toute ma gratitude va à Alan Bryden ainsi quà ses collègues du DCAF pour leurs remarques pertinentes et leur soutien rédactionnel. Enfin, merci à tous les officiers de police de haut rang grâce à qui j’ai pu préciser ma pensée. Je leur suis très reconnaissant.

2 Voir par exemple, la polémique au sujet de la politique de recrutement des SPG: ceux-ci ont convaincu le Conseil de police de leur capacité à enquêter sur eux-mêmes en créant une équipe spéciale (Salia 2015:32-33). Voir également le Daily Graphic (2015a; 2015b; 2015c).

3 Pour une discussion sur l'évolution historique des SPG, voir Aning (2002).

4 Voir aussi Aning (2008a).

${ }^{5}$ Le Conseil national de libération, administré conjointement par des militaires et des policiers, était dirigé par le lieutenant-général Joseph Ankrah. Faisaient également parti du conseil : le commissaire de police JWK Harley, le directeur adjoint colonel EK Kotoka, l'officier général commandant les forces armées ghanéennes BA Yakubu, le commissaire colonel AK Ocran, le sous-commissaire JEO Nunoo, le major AA Afrifa et le commissaire adjoint AK Deku (Barker 1979: 177-179).

${ }^{6}$ D'autres règlementations qui ont régi les SPG sont par exemple: la loi de 1963 sur les services de sécurité (loi no 202), la loi de 1965 sur les services de police (loi no 284), le chapitre XIII de la Constitution de 1969 de la République du Ghana, la loi de 1970 sur les services de police (loi no 350), le décret (amendement) de 1974 sur les forces de police, le chapitre XVII, art. 172-175 de la Constitution de 1979 de la République of Ghana et la IVe Constitution républicaine de 1992.

7 Voir à ce propos les dispositions pertinentes figurant dans la Constitution de la République of Ghana (1992: art. 203[10]).

${ }^{8}$ Loi sur les services de police (1970: art. 36[1]). 
9 Voir à ce propos la loi sur les services de police (1970: art. 10-16). Boyes (1971: 241-243) se réfère à cet article dans son rapport de 1971.

10 Voir l'article 203 (2-3) de la Constitution de 1992. Voir également la loi no 350, 3e partie, art. 10-16. Pour comprendre le lien avec le rapport Boyes de 1971, voir Boyes (1971 : 24-26). L'article 201 de la Constitution de 1992 place le vice-président à la tête des Conseils de police, des forces armées et des prisons. Cependant, la Constitution de 1992 a été amendée en 1996 par la loi no 527 afin d'anticiper la situation dans laquelle un président et un vice-président issus de partis différents et réunis dans un même gouvernement ne pourraient pas résoudre leurs désaccords (Quantson 2000: 292-308).

11 Lors de plusieurs entretiens avec des officiers de police de haut rang, il a été fait mention d'une personnalité nommée pour des raisons politiques pendant l'époque révolutionnaire (1981-1992) qui a contribué à saper la légitimité des procédures de recrutement en plaçant à certains postes des membres de son parti. Entretien avec l'auteur à Accra, le 7 mars 2015.

12 Pour une discussion approfondie de ces processus, voir Aning et Lartey (2008). Ces réformes ont été appelées « réforme du secteur public», « renouveau institutionnel national», "réforme pour l'ordre public» ou encore «réforme pour l'Etat de droit». Elles ont eu lieu dans les années 80 dans le contexte du Programme pour le redressement économique et du Programme de redressement structurel. Le sous-programme « Renouveau institutionnel national» a été configuré pour mettre en œuvre la réforme de plusieurs institutions ghanéennes. Voir également Atuguba (2007).

13 Ces obligations sont bien connues, excepté en ce qui concerne le contrôle disciplinaire: elles peuvent être considérées comme un renforcement des devoirs du Conseil de police de 1969, comportant un élargissement du mandat initial.

14 Cette institution a été créée dans le cadre de la Règlementation des services de police $(2012$ : rég. 8,10$)$.

15 Entretien avec un officier supérieur de police, Accra, le 10 mars 2015.

16 Voir à ce propos l'article correspondant de la Constitution de la République du Ghana (1979: Ch. XVII, art. 173[1a]).

17 Voir, par exemple, Avuyi (1995): officier d'état-major adjoint (1990). Voir aussi l'entrevue du 11 mars 2015 à Accra. D’après l'officier interviewé, le subalterne avait été nommé au conseil pour «compléter l'effectif».

18 Lors d'un entretien avec l'ancien vice-président, puis président, John AttahMills, il a expliqué en détail pourquoi la scission entre le président Rawlings et son vice-président avait posé des problèmes si délicats: ce dernier avait quitté officiellement la coalition gouvernementale pour diriger une autre faction du parti, tout en conservant son statut de vice-président.

19 Voir Ghana Web (2013). D’après le rapport, «le lundi 11 novembre 2013, le président John Dramani Mahama a nommé le vice-président Paa Kwesi 
Bekoe Amissah-Arthur à la tête d'un nouveau Conseil de police. Les membres sont: le ministre de l'Intérieur Kwesi Ahwoi, l'inspecteur général de police Mohammed Alhassan, le procureur général adjoint Dominic Ayine, la commissaire Rose Atinga Bio, le commissaire Kwasi Nkansah (Rtd.), l'inspecteur Charles Obiri Yeboah, Nancy Amarteifio, le révérend Lartey Lawson et Alhaji Salifu Osman.»

${ }^{20}$ Entretiens avec plusieurs intervenants de janvier à septembre 2014 et de janvier à mars 2015 .

21 Pendant les recherches qui ont abouti à la rédaction de ce chapitre, l'un des problèmes les plus épineux a été l'ingérence constante du pouvoir exécutif dans le fonctionnement du Conseil de police. Comme l'a rétorqué un officier "vérifiez si un nouveau Conseil de police avait été formé par le président Kufuor (2000-2008) avant l'annulation de la reconduction des contrats. Si la réponse est non, le conseil a-t-il bien réfléchi avant d’avaliser ces soi-disant reconductions, que le président lui-même a dû invalider? Il $\mathrm{y}$ a eu une autre incohérence de la part des dirigeants politiques car le gouvernement Kufuor a prolongé l'affectation de certains officiers sous couvert de contrats. Bien entendu, ces contrats ont été résiliés ou non reconduits lorsque le président John Mills (2009-2012) a accédé à la présidence.». Entretien téléphonique, Accra, le 10 mars 2015.

${ }^{22}$ Entretien, Accra, le 8 mars 2015.

23 Pour un autre point de vue, voir Assamoah (2104).

24 Entretien, Accra, le 13 décembre 2015.

25 Voir le «Daily Guide News », édition du mardi 23 octobre 2014.

${ }^{26}$ Il faudrait mandater une étude pour évaluer l'impact et l'efficacité de cette stratégie ostensible afin d’en examiner les conséquences. Le taux de criminalité a-t-il diminué? La police est-elle devenue plus efficace pour autant? Les moyens nécessaires pour atteindre ce niveau de présence pourraient-ils être alloués à des postes ou à des circonscriptions chargés de maintenir la sécurité sur leur propre territoire? Que font les équipes déployées dans les rues que les postes (s'ils sont bien équipés) ne puissent pas faire? Une personne interrogée à ce sujet a déclaré "qu'il ne s'agissait pas d'abus ni d'un désir de gagner la sympathie du public mais plutôt d'un manque de ressources» Entretien du 15 mars 2015 à Accra.

27 Entretien avec un officier supérieur de police, Accra, le 8 mars 2015. 